\title{
Evidence from animal studies supports the "entheseal stress' hypothesis of ankylosing spondylitis
}

The results of experiments carried out in mice could provide new insight into the relationship between inflammation and ankylosis in spondyloarthritis (SpA). In a model of spontaneous arthritis, treatment with glucocorticoids inhibited inflammation but did not halt the formation of new bone that leads to ankylosis in SpA. In addition, gene expression analysis of the paws of affected mice suggest that bone morphogenetic protein (BMP) signaling is a key mediator in both inflammation and bone formation.

\section{4 ...inflammation and new} bone formation are probably linked but independent events... 77

The study, led by Rik Lories of KU Leuven, lends support to the hypothesis that inflammation and new bone formation are probably linked but independent events, which could both arise from a common noninflammatory trigger.

The team of investigators looked at the early stages of the spontaneous arthritis model, which is characterized by ankylosing enthesitis and short-lived (rather than chronic) inflammation. Daily treatment of male DBA/1 mice with dexamethasone $(0.5 \mathrm{mg}$ per $\mathrm{kg}$ body weight) inhibited inflammation compared with placebo-treated mice, as assessed by repeated PET imaging, whereas dexamethasone treatment resulted in no difference in the severity of ankylosis between the two groups of mice. In vivo dual energy X-ray absorptiometry showed that, as expected, glucocorticoid treatment reduced global bone density in the mice.

As Lories puts it, these results "exemplify the spondyloarthritis bone paradox in which trabecular bone is lost while new bone formation is taking place at the margins of the same bones. This is an amazing paradox also seen in patients with spondyloarthritis."

In further experiments in the study, ex vivo gene expression analysis of mouse paws demonstrated that neutrophil chemokines were upregulated in the early phase of disease, and in vitro stimulation with BMPs upregulated neutrophil chemokines in human progenitor cells; the latter effect was inhibited by dexamethasone. "This suggests that activation of BMP signaling may contribute to both ankylosis and inflammation in this model," adds Lories.

Sarah Onuora

Original article Braem, K. et al. Inhibition of inflammation but not ankylosis by glucocorticoids in mice: further evidence for the entheseal stress hypothesis. Arthritis Res. Ther. 14, R59 (2012)

Further reading Lories, $\mathrm{R}$. The balance of tissue repair and remodeling in chronic arthritis. Nat. Rev. Rheumatol. 7, 700-707 (2011) 